\title{
О МАТРИЧНОЙ КОРРЕКЦИИ ДВОЙСТВЕННОЙ ПАРЫ НЕСОБСТВЕННЫХ ЗАДАЧ ЛИНЕЙНОГО ПРОГРАММИРОВАНИЯ ПО МИНИМУМУ ВЗВЕШЕННОЙ ЕВКЛИДОВОЙ МАТРИЧНОЙ НОРМЫ
}

\author{
В. И. Ерохин ${ }^{\star}$ В. В. Волков ${ }^{\star *}$, М. Н. Хвостов ${ }^{\star *}$ \\ ${ }^{*}$ Военно-космическая академия имени А. Ф. Можайского, \\ **Борисоглебский билиал Воронежского государственного университета
}

\section{Поступила в редакцию 15.07.2019 г.}

\begin{abstract}
Аннотация. В статье рассматривается матричная коррекция двойственной пары несобственных задач линейного программирования с минимальной взвешенной евклидовой нормой. Взвешивание обеспечивается путем умножения расширенной матрицы коррекции слева и справа на невырожденные матрицы. Основной целью взвешивания является включение в задачу линеного программирования сведений о трудоемкости коррекции расширенной матрицы системы ограничений. Под матричной коррекцией в данной статье подразумевается изменение (коррекция) любых коэффициентов системы ограничений с целью обеспечения ее совместности. Указанная проблема сведена к вспомогательной задаче безусловной дифференцируемой минимизации. Обоснованием этого перехода является представленая в статье теорема об оптимальной по минимуму взвешенной евклидовой нормы коррекции двойственной пары несобственных задач линейного программирования. Данная теорема является следствием теоремы о существовании решения задачи коррекции расширенной матрицы ограничений двойственной пары несобственных задач линейного программирования по минимуму взвешенной евклидовой нормы. В свою очередь, последняя теорема базируется на теореме о матричном решении обратной задачи линейного программирования. Формулировки последних теорем также приводятся в статье. В качестве возможного инструмента численного решения данной задачи рассмотрен квазиньютоновский алгоритм Бройдена-Флетчера-Голдфарба-Шанно. Рассматривается задача поиска расширенной матрицы коррекции, минимальной по взвешенной евклидовой норме. Данная задача определяется следующими параметрами: расширенной матрицей системы ограничений, невырожденными весовыми матрицами и начальным приближением. Решение представлено аргументом целевой функции и ее значением. Приведены результаты вычислительных экспериментов по исследованию сходимости предложенного алгоритма по целевой функции и по аргументу.

Ключевые слова: линейное программирование, несобственная задача линейного программирования, двойственная пара задач линейного программирования, матричная коррекция, взвешенная евклидова норма.
\end{abstract}

\section{ВВЕДЕНИЕ}

Линейные оптимизационные модели, формализованные в виде задач линейного программирования (ЛП) $[1,2]$, широко применяются в различных областях науки, техники и экономики. Рассмотрим задачу ЛП в канонической форме:

(с) Ерохин В. И., Волков В. В., Хвостов М. Н., 2019

$$
L(A, b, c): A x=b, x \geq 0, c^{\mathrm{T}} x \rightarrow \max ,
$$

где $A \in \mathrm{R}^{m \times n}, \quad c, x \in \mathrm{R}^{n}, \quad b \in \mathrm{R}^{m}, \quad \mathcal{X}(A, b)=$ $=\{x \mid A x=b, x \geq 0\}$ - ее допустимое множество. Задачи ЛП часто являются неразрешимыми [3-5]. Пусть, например, система ограничений задачи (1) несовместна, т. е. $\mathcal{X}(A, b)=\varnothing$, и необходимо такое ее изменение (коррекция), которая приведет ее к собственной задаче линейного программирования, в некотором смысле «близкой» к исходной [6-8]. 


\section{В. И. Ерохин, В. В. Волков, М. Н. Хвостов}

В случае, когда подобная коррекция предполагает изменение (коррекцию) любых коэффициентов как левых, так и правых частей уравнений и неравенств ограничений задачи ЛП, принято говорить о матричной коррекции задач ЛП [9-10]:

$$
P(A, b):\left\{\begin{array}{l}
\|[H-h]\| \rightarrow \min , \\
\mathcal{X}(A+H, b+h) \neq \varnothing,
\end{array}\right.
$$

где $\|\cdot\|$ - некоторая матричная норма.

Часто возникает необходимость включить в модель (2) дополнительные данные, характеризующие (в прикладной задаче) трудоемкость или стоимость изменения отдельных ее коэффициентов. Одним из наиболее известных способов является применение взвешенной евклидовой нормы, а одним из наиболее распространенных вариантов взвешивания является взвешивание с помощью левого и правого умножения на невырожденные матрицы [11]:

$$
Z^{L R}(A, b):\left\{\begin{array}{l}
\|L \cdot[H-h] \cdot R\| \rightarrow \min \\
\mathcal{X}(A+H, b+h) \neq \varnothing
\end{array}\right.
$$

где $L \in \mathrm{R}^{m \times m}, R \in \mathrm{R}^{(n+1) \times(n+1)}, L, R$ - невырожденные, причем $R_{i j} \geq 0, \quad R_{i j}^{-1} \geq 0, \quad i \in 1, \ldots, n+1$, $j \in 1, \ldots, n+1$.

Еще одним способом взвешивания является умножение по Адамару матрицы коррекции на матрицу с положительными коэффициентами [12]:

$$
Z^{W}(A, b):\left\{\begin{array}{l}
\|W \circ[H-h]\| \rightarrow \min , \\
\mathcal{X}(A+H, b+h) \neq \varnothing,
\end{array}\right.
$$

где $W \in \mathrm{R}^{m \times(n+1)}, W_{i j}>0, i \in 1, \ldots, m, j \in 1, \ldots, n+1$.

Следует заметить, что коррекция системы ограничений задачи линейного программирования, делающая ее допустимую область непустой, еще не гарантирует, что задача линейного программирования станет собственной. Указанное обстоятельство послужило мотивацией для развития методов коррекции несобственных задач ЛП, существенно использующих теорию двойственности [1315] и, в силу этого, гарантирующих собственность скорректированных задач - прямой (вида (1)), и двойственной:

$$
L^{*}(A, b, c): u^{\mathrm{T}} A \geq c^{\mathrm{T}}, b^{\mathrm{T}} u \rightarrow \min ,
$$

где $u \in \mathrm{R}^{m}$. Задача (3) явяляется задачей ЛП в основной форме, $\mathcal{U}(A, c)=\left\{u \mid u^{\mathrm{T}} A \geq c^{\mathrm{T}}\right\}-$ ее допустимое множество.

Коррекция допустимых множеств прямой (1) и двойственной задачи ЛП (3) [16-17] может быть сформулирована, например, в виде следующей задачи:

$$
D(A, b, c):\left\{\begin{array}{l}
\|[H-h]\| \rightarrow \min , \\
(A+H) x=(b+h), x \geq 0, \\
u^{\mathrm{T}}(A+H) x \geq c^{\mathrm{T}}, \\
c^{\mathrm{T}} x=(b+h)^{\mathrm{T}} u .
\end{array}\right.
$$

Мы будем рассматривать модификацию задачи (4) - задачу взвешенной матричной коррекции двойственной пары несобственных задач ЛП (матричной коррекции двойственной пары несобственных задач ЛП по минимуму взвешенной евклидовой матричной нормы):

$$
D^{L R}(A, b, c):\left\{\begin{array}{l}
\|L \cdot[H-h] \cdot R\| \rightarrow \min , \\
(A+H) x=(b+h), x \geq 0, \\
u^{\mathrm{T}}(A+H) x \geq c^{\mathrm{T}}, \\
c^{\mathrm{T}} x=(b+h)^{\mathrm{T}} u .
\end{array}\right.
$$

\section{МАТЕРИАЛЫ И МЕТОДЫ ИССЛЕДОВАНИЯ}

Докажем некоторые вспомогательные утверждения. Первое утверждение представляет собой модификацию теоремы о матричном решении обратной задачи ЛП [16].

Теорема 1. Пусть весовые коэффициенты заданы невырожденными матрицами $L \in \mathrm{R}^{m \times m}, R \in \mathrm{R}^{(n+1) \times(n+1)}$, причем $R_{i j} \geq 0, R_{i j}^{-1} \geq 0$, $i \in 1, \ldots, n+1, \quad j \in 1, \ldots, n+1$. Тогда семейство матриц, гарантирующих, что при заданных $b \in \mathrm{R}^{m}$ и $c \in \mathrm{R}^{n}$ вектор $\bar{x} \in \mathrm{R}^{n}, \bar{x} \geq 0$ принадлежит множеству решений задачи ЛП $L(A, b, c)$, а вектор $\bar{u} \in \mathrm{R}^{m}, \bar{u} \neq 0$ принадлежит множеству решений задачи ЛП $L^{*}(A, b, c)$. существует тогда и только тогда, когда выполняется условие

$$
c^{\mathrm{T}} \bar{x}=b^{\mathrm{T}} \bar{u}=\alpha .
$$

и описывается формулой

$$
A=\hat{A}+\Delta A \text {, }
$$


О матричной коррекции двойственной пары несобственных задач тинейного программирования ...

$$
\begin{gathered}
\hat{A}=\frac{b \bar{x}^{\mathrm{T}}\left(R R^{\mathrm{T}}\right)^{-1}}{\bar{x}^{\mathrm{T}}\left(R R^{\mathrm{T}}\right)^{-1} \bar{x}}+\frac{\left(L^{\mathrm{T}} L\right)^{-1} \bar{u} d^{\mathrm{T}}}{\bar{u}^{\mathrm{T}}\left(L^{\mathrm{T}} L\right)^{-1} \bar{u}}- \\
-\alpha \frac{\left(L^{\mathrm{T}} L\right)^{-1} \overline{u x}^{\mathrm{T}}\left(R R^{\mathrm{T}}\right)^{-1}}{\bar{u}^{\mathrm{T}}\left(L^{\mathrm{T}} L\right)^{-1} \overline{u x}^{\mathrm{T}}\left(R R^{\mathrm{T}}\right)^{-1} \bar{x}}
\end{gathered}
$$

- матрица с минимальной взвешенной евклидовой нормой,

$$
\begin{gathered}
d=\left[d_{1}, \ldots, d_{n}\right]^{\mathrm{T}}, \\
d_{i}=\left\{\begin{array}{l}
0, \text { если } c_{i} \leq 0 u \bar{x}_{i}=0, \\
c_{i}, \text { в противном случае, }
\end{array}\right.
\end{gathered}
$$

$\triangle A$ - произвольная матрица такая, что

$$
\bar{u}^{\mathrm{T}} \Delta A=0, \quad \Delta A \bar{x}=0 .
$$

При этом

$$
\begin{gathered}
\|L \hat{A} R\|^{2}=\frac{\|L b\|^{2}}{\left\|R^{-1} \bar{x}\right\|^{2}}+\frac{\left\|d^{\mathrm{T}} R\right\|^{2}}{\left\|\bar{u}^{T} L^{-1}\right\|^{2}}- \\
-\frac{\alpha^{2}}{\left\|R^{-1} \bar{x}\right\|^{2}\left\|\bar{u}^{T} L^{-1}\right\|^{2}},
\end{gathered}
$$

где $\|\cdot\|$ - в зависимости от контекста, евклидова матричная или векторная норма.

Следующее утверждение представляет собой модификацию теоремы существования решения задачи коррекции расширенной матрицы ограничений двойственной пары несобственных задач ЛП по минимуму евклидовой нормы.

Теорема 2. Пусть весовые коэффициенты заданы невырожденными матрицами $L \in \mathrm{R}^{m \times m}$, $R \in \mathrm{R}^{(n+1) \times(n+1)}, \quad$ причем $\quad R_{i j} \geq 0, \quad R_{i j}^{-1} \geq 0$, $i \in 1, \ldots, n+1, \quad j \in 1, \ldots, n+1$. Тогда семейство матриц, корректирующих двойственную пару несобственных задач ЛП $L(A, b, c)$ и $L^{*}(A, b, c)$ при заданных ненулевых решениях с учетом весовых коэффициентов $L$ и $R$, т. е. гарантирующих, что вектор $\bar{x} \in \mathrm{R}^{n}, \bar{x} \geq 0$ принадлежит множеству решений задачи ЛП $L(A+H, b+h, c)$, а вектор $\bar{u} \in \mathrm{R}^{m}, \bar{u} \neq 0$ принадлежит множеству решений задачи ЛП $L^{*}(A+H, b+h, c)$, имеет вид

$$
[H-h]=[\hat{H}-\hat{h}]+[\Delta H-\Delta h],
$$

где $H, \hat{H}, \Delta H \in \mathrm{R}^{m \times n}, h, \hat{h}, \Delta h \in \mathrm{R}^{m},[\hat{H}-\hat{h}]-$ матрица с минимальной взвешенной евклидовой нормой,

$$
\begin{aligned}
& {\left[\begin{array}{ll}
\hat{H} & -\hat{h}
\end{array}\right]=\frac{(b-A \bar{x})\left[\begin{array}{ll}
\bar{x}^{\mathrm{T}} & 1
\end{array}\right]\left(R R^{\mathrm{T}}\right)^{-1}}{\left[\begin{array}{ll}
\bar{x}^{\mathrm{T}} & 1
\end{array}\right]\left(R R^{\mathrm{T}}\right)^{-1}\left[\begin{array}{ll}
\bar{x}^{\mathrm{T}} & 1
\end{array}\right]^{\mathrm{T}}}+} \\
& +\frac{\left(L^{\mathrm{T}} L\right)^{-1} \bar{u}\left[d^{\mathrm{T}} b^{\mathrm{T}} \bar{u}-c^{\mathrm{T}} \bar{x}\right]}{\bar{u}^{\mathrm{T}}\left(L^{\mathrm{T}} L\right)^{-1} \bar{u}}- \\
& -\alpha \frac{\left(L^{\mathrm{T}} L\right)^{-1} \bar{u}\left[\begin{array}{ll}
\bar{x}^{\mathrm{T}} & 1
\end{array}\right]\left(R R^{\mathrm{T}}\right)^{-1}}{\left[\begin{array}{ll}
\bar{x}^{\mathrm{T}} & 1
\end{array}\right]\left(R R^{\mathrm{T}}\right)^{-1}\left[\begin{array}{ll}
\bar{x}^{\mathrm{T}} & 1
\end{array}\right]^{\mathrm{T}} \bar{u}^{\mathrm{T}}\left(L^{\mathrm{T}} L\right)^{-1} \bar{u}}, \\
& \alpha=b^{\mathrm{T}} \bar{u}-\bar{u}^{\mathrm{T}} A \bar{x}, \\
& d=\left[d_{1}, \ldots, d_{n}\right]^{\mathrm{T}}, \\
& d_{i}=\left\{\begin{array}{l}
0, \text { если }\left(c-A^{\mathrm{T}} \bar{u}\right)_{i} \leq 0 \text { и } \bar{x}_{i}=0, \\
\left(c-A^{\mathrm{T}} \bar{u}\right)_{i}, \text { в противном случае, }
\end{array}\right.
\end{aligned}
$$

$[\Delta H-\Delta h]$ - произвольная матрица такая,

что

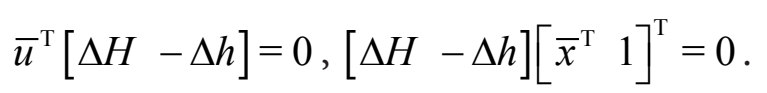

При этом

$$
\begin{gathered}
\|L[\hat{H}-\hat{h}] R\|^{2}=\frac{\|L(b-A \bar{x})\|^{2}}{\left\|R^{-1}\left[\begin{array}{ll}
\bar{x}^{\mathrm{T}} & 1
\end{array}\right]^{\mathrm{T}}\right\|^{2}}+ \\
+\frac{\left\|\left[d^{\mathrm{T}} b^{\mathrm{T}} \bar{u}-c^{\mathrm{T}} \bar{x}\right] R\right\|^{2}}{\left\|\bar{u}^{T} L^{-1}\right\|^{2}}- \\
-\frac{\alpha^{2}}{\left\|R^{-1}\left[\begin{array}{ll}
\bar{x}^{\mathrm{T}} & 1
\end{array}\right]^{\mathrm{T}}\right\|^{2}\left\|\bar{u}^{T} L^{-1}\right\|^{2}} .
\end{gathered}
$$

Базируясь на результатах, представленных в предыдущих теоремах, сформулируем теорему об оптимальной по минимуму взвешенной евклидовой нормы коррекции двойственной пары несобственных задач ЛП.

Теорема 3. Задача $D^{L R}(A, b, c)$ эквивалентна задаче безусловной минимизации функции

$$
\Phi^{H h}(\tilde{x}, \tilde{u})=\|L[H-h] R\|^{2} \rightarrow \min _{\tilde{x}, \tilde{u}}
$$

где

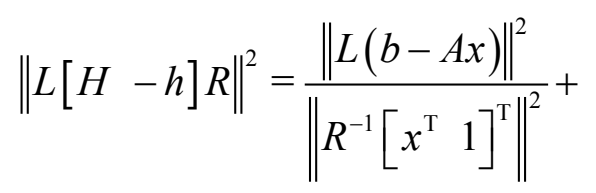




$$
\begin{gathered}
+\frac{\left\|\left[d^{\mathrm{T}} b^{\mathrm{T}} u-c^{\mathrm{T}} x\right] R\right\|^{2}}{\left\|u^{\mathrm{T}} L^{-1}\right\|^{2}}- \\
-\frac{\alpha^{2}}{\left\|R^{-1}\left[\begin{array}{ll}
x^{\mathrm{T}} & 1
\end{array}\right]^{\mathrm{T}}\right\|^{2}\left\|u^{T} L^{-1}\right\|^{2}}, \\
x=x(\tilde{x})=\left[\begin{array}{c}
\tilde{x}_{1}^{2} \\
\vdots \\
\tilde{x}_{n}^{2}
\end{array}\right]=\operatorname{diag}(\tilde{x}) \tilde{x}, u=u(\tilde{u})=\tilde{u}, \\
d_{i}=\left\{\begin{array}{l}
0, \text { eсли }\left(c-A^{\mathrm{T}} u\right)_{i} \leq 0 \text { u } x_{i}=0, \\
\left(c-A^{\mathrm{T}} u\right)_{i}, \text { в противном случае. }
\end{array}\right.
\end{gathered}
$$

При этом, если векторы $\tilde{x}^{*}$ и $\tilde{u}^{*}$ являются решением задачи (5) и

$$
\Phi^{H h}\left(\tilde{x}^{*}, \tilde{u}^{*}\right)>0
$$

то единственное решение задачи $D^{L R}(A, b, c)$, связанное с указанными векторами, выражается формулой

$$
\begin{gathered}
{\left[H^{*}-h^{*}\right]=\frac{\left(b-A x^{*}\right)\left[\begin{array}{c}
x^{*} \\
1
\end{array}\right]^{\mathrm{T}}\left(R R^{\mathrm{T}}\right)^{-1}}{\left[\begin{array}{c}
x^{*} \\
1
\end{array}\right]^{\mathrm{T}}\left(R R^{\mathrm{T}}\right)^{-1}\left[\begin{array}{c}
\left.x^{*}\right] \\
1
\end{array}\right]}+} \\
+\frac{\left(L^{\mathrm{T}} L\right)^{-1} u^{*}\left[d^{\mathrm{T}} b^{\mathrm{T}} u^{*}-c^{\mathrm{T}} x^{*}\right]}{u^{* \mathrm{~T}}\left(L^{\mathrm{T}} L\right)^{-1} u^{*}}- \\
-\alpha \frac{\left(L^{\mathrm{T}} L\right)^{-1} u^{*}\left[\begin{array}{c}
x^{*} \\
1
\end{array}\right]^{\mathrm{T}}\left(R R^{\mathrm{T}}\right)^{-1}}{\left[\begin{array}{c}
x^{*} \\
1
\end{array}\right]^{\mathrm{T}}\left(R R^{\mathrm{T}}\right)^{-1}\left[x^{*}\right] u^{* \mathrm{~T}}\left(L^{\mathrm{T}} L\right)^{-1} u^{*}}
\end{gathered}
$$

где векторы $x^{*}=x\left(\tilde{x}^{*}\right), u^{*}=u\left(\tilde{u}^{*}\right), d=d\left(\tilde{x}^{*}, \tilde{u}^{*}\right)$. Векторы $x^{*}, u^{*}$ являются решением скорректированных задач.

Замечание. Доказательства теоремы 1-3 аналогичны доказательствам соответствующих теорем без взвешивания $[14,18]$, с учетом замены $x$ на $R x$ и $u$ на $u L$.

\section{РЕЗУЛЬТАТЫ ИССЛЕДОВАНИЙ И ИХ ОБСУЖДЕНИЕ}

В качестве примера рассмотрим задачу поиска расширенной матрицы коррекции, минимальной по взвешенной евклидовой норме, со следующими параметрами:

$$
\begin{aligned}
& A=\left[\begin{array}{rrrrrrrrrr}
1 & 0 & 0 & -1 & 0 & 0 & 0 & 1 & 0 & 0 \\
0 & 1 & 0 & 0 & -1 & 0 & 0 & 0 & 1 & 0 \\
0 & 0 & 1 & 0 & 0 & -1 & 0 & 0 & 0 & 1 \\
0 & 0 & 0 & 1 & 0 & 0 & -1 & 0 & 0 & 0 \\
0 & 0 & 0 & 0 & 1 & 0 & 0 & -1 & 0 & 0 \\
0 & 0 & 0 & 0 & 0 & 1 & 0 & 0 & -1 & 0 \\
0 & 0 & 0 & 0 & 0 & 0 & -1 & 0 & 0 & -1
\end{array}\right], \\
& b^{\mathrm{T}}=\left[\begin{array}{lllllll}
-4 & 2 & 1 & 0 & -3 & 1 & 2
\end{array}\right], \\
& c^{\mathrm{T}}=\left[\begin{array}{llllllllll}
-1 & -2 & -1 & 5 & -1 & 2 & 0 & -1 & 4 & -1
\end{array}\right] \text {. }
\end{aligned}
$$

Весовые коэфициенты заданы следующими матрицами:

$$
\begin{aligned}
L & =\left[\begin{array}{lllllll}
1 & 0 & 0 & 0 & 0 & 0 & 0 \\
0 & 2 & 0 & 0 & 0 & 0 & 0 \\
0 & 0 & 3 & 0 & 0 & 0 & 0 \\
0 & 0 & 0 & 4 & 0 & 0 & 0 \\
0 & 0 & 0 & 0 & 5 & 0 & 0 \\
0 & 0 & 0 & 0 & 0 & 6 & 0 \\
0 & 0 & 0 & 0 & 0 & 0 & 7
\end{array}\right], \\
R & =\left[\begin{array}{lllllllllll}
1 & 0 & 0 & 0 & 0 & 0 & 0 & 0 & 0 & 0 & 0 \\
0 & 1 & 0 & 0 & 0 & 0 & 0 & 0 & 0 & 0 & 0 \\
0 & 0 & 2 & 0 & 0 & 0 & 0 & 0 & 0 & 0 & 0 \\
0 & 0 & 0 & 2 & 0 & 0 & 0 & 0 & 0 & 0 & 0 \\
0 & 0 & 0 & 0 & 3 & 0 & 0 & 0 & 0 & 0 & 0 \\
0 & 0 & 0 & 0 & 0 & 3 & 0 & 0 & 0 & 0 & 0 \\
0 & 0 & 0 & 0 & 0 & 0 & 2 & 0 & 0 & 0 & 0 \\
0 & 0 & 0 & 0 & 0 & 0 & 0 & 2 & 0 & 0 & 0 \\
0 & 0 & 0 & 0 & 0 & 0 & 0 & 0 & 1 & 0 & 0 \\
0 & 0 & 0 & 0 & 0 & 0 & 0 & 0 & 0 & 1 & 0 \\
0 & 0 & 0 & 0 & 0 & 0 & 0 & 0 & 0 & 0 & 5
\end{array}\right] .
\end{aligned}
$$

В качестве начального приближения алгоритма выбраны векторы:

$$
\tilde{x}^{0}=A^{+} b, \tilde{u}^{0}=A^{+\mathrm{T}} c .
$$


О матричной коррекции двойственной пары несобственных задач линейного программирования ...

Алгоритм Бройдена-Флетчера-Голдфарба-Шанно, модифицированный для минимизации целевой функции (5) дает следующее решение:

$$
x^{*}=\left[\begin{array}{r}
693,56915735 \\
0,01477895 \\
195,51455872 \\
12,10965030 \\
82,39024295 \\
171,38480932 \\
0,03091958 \\
65,22099479 \\
167,99684095 \\
0,68166074
\end{array}\right], u^{*}=\left[\begin{array}{r}
-7,46329866 \\
-3,32545906 \\
-2,13060861 \\
-1,92138317 \\
-5,01308303 \\
-0,85468452 \\
1,32141450
\end{array}\right] .
$$

Следует заметить, что функция $\Phi^{H h}(\tilde{x}, \tilde{u})$ не является всюду дифференцируемой из-за того, что элементы вектора $d$ негладко зависят от элементов векторов $x$ и $u$. Но, несмотря на то, что на то, что минимизуремая функция не является дифференцируемой для всех значений $x, u$ (и, как следствие, представленные выше формулы для вычисления производных обоснованы не для всех значений $x, u)$, процесс вычислений протекает без сбоев и остановок.

Значение
$\Phi^{H h}\left(x^{*}, u^{*}\right)=\left\|L\left[H^{*}-h^{*}\right] R\right\|^{2}=1,933706$, ев-

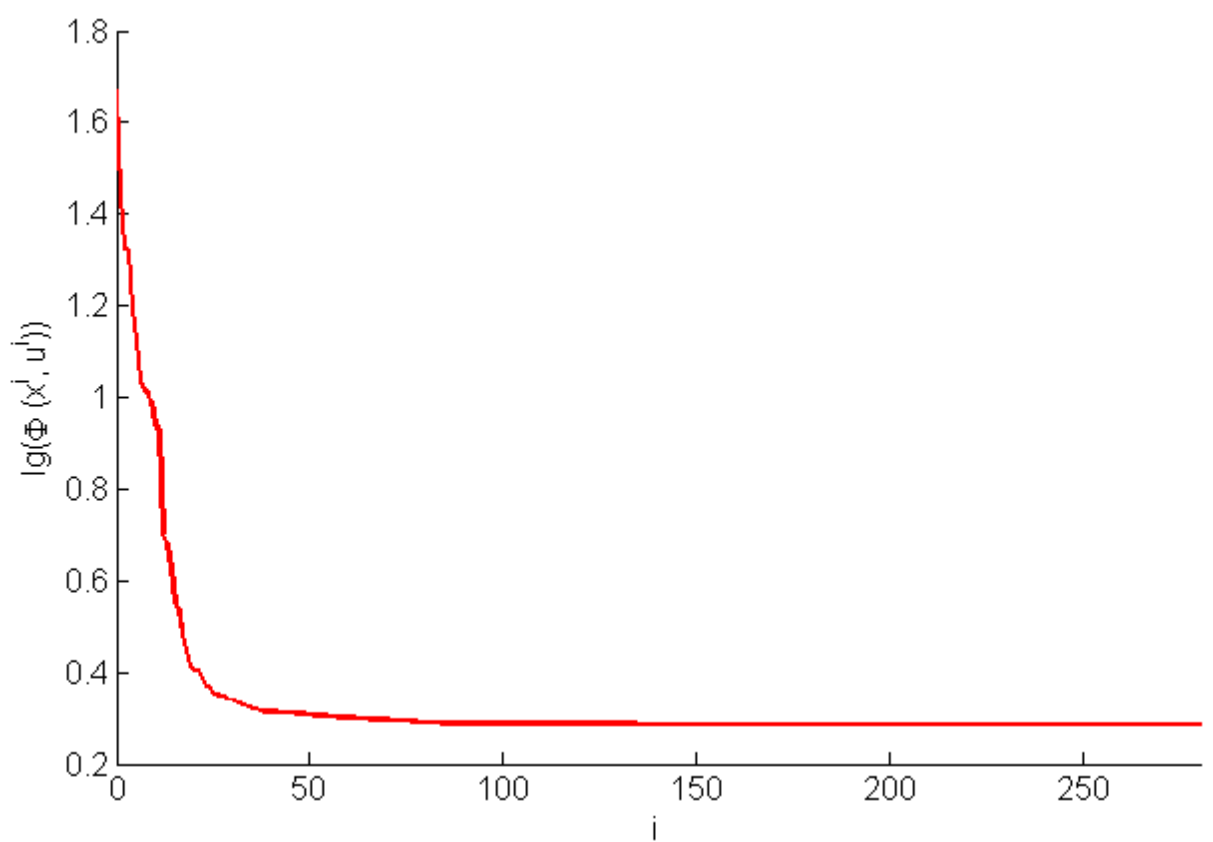

Рис. 1. График сходимости иелевой функиии клидова матричная норма расширенной матницы коррекции $\left\|\left[H^{*}-h^{*}\right]\right\|=1,225013$.

$$
\begin{aligned}
& \text { Проверка показывает, } \\
& \left\|\left(A+H^{*}\right) x^{*}-\left(b+h^{*}\right)\right\|=1,689350 \cdot 10^{-13} \text {, } \\
& \left\|u^{* \mathrm{~T}}\left(A+H^{*}\right)-c^{\mathrm{T}}\right\|=2,627267 \cdot 10^{-15} \text {. }
\end{aligned}
$$
что

\section{ЗАКЛЮЧЕНИЕ}

Решение задачи матричной коррекции двойственной пары несобственных задач ЛП по минимуму взвешенной евклидовой матричной нормы $D^{L R}(A, b, c)$ базируется не только на теории двойственности но и предполагает применение взвешенной евклидовой нормы. Так, использование теории двойственности позволяет гарантировать собственность скорректированных прямой и двойственных задач ЛП. В свою очередь, применение взвешенной евклидовой нормы позволяет включить в линейную оптимазационную модель дополнительные данные, которые могут определять трудоемкость изменения отдельных коэффициентов системы ограничений данной модели.

Представленная в статье теорема об оптимальной по минимуму взвешенной евклидовой нормы коррекции двойственной пары несобственных задач ЛП (теорема 3) позволяет свести задачу $D^{L R}(A, b, c)$ к задаче без- 


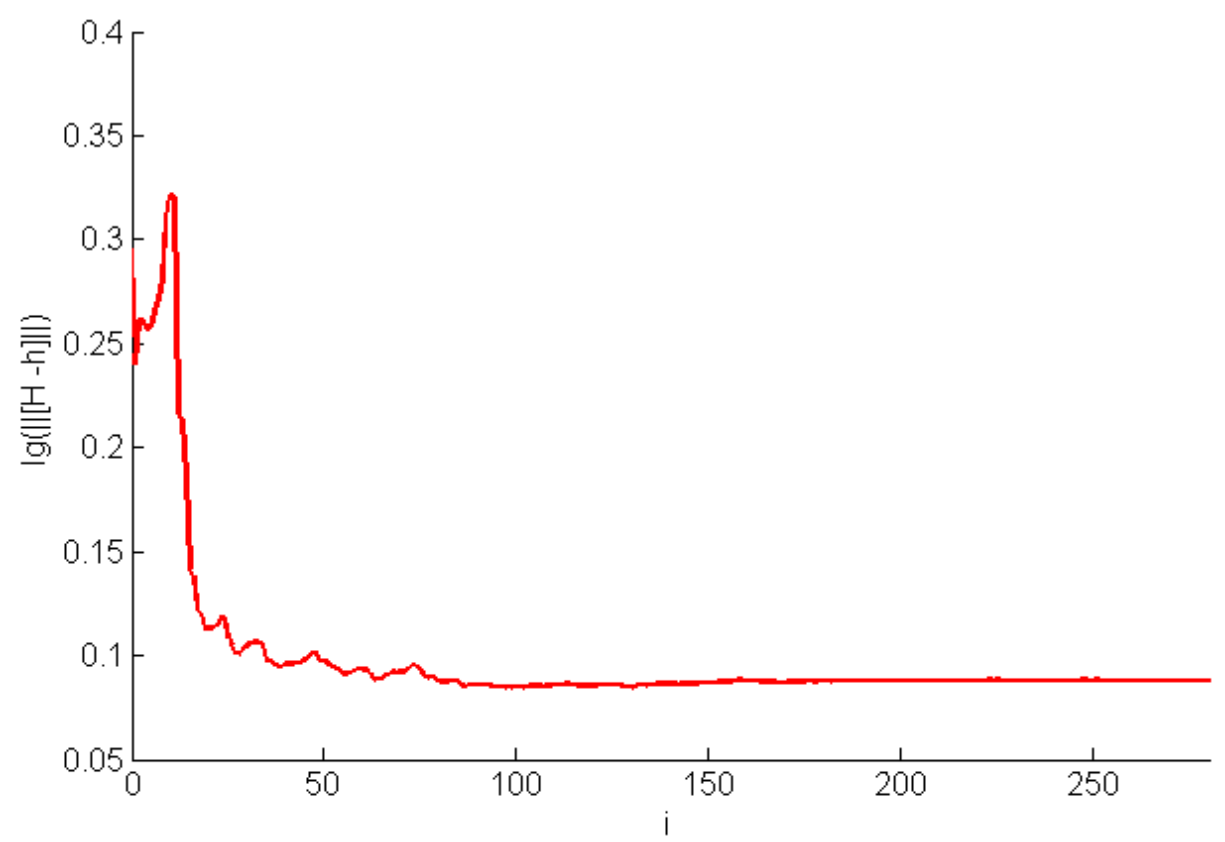

Рис. 2. График сходимости аргумента

условной минимизации функции $\Phi^{H h}(\tilde{x}, \tilde{u})$. Для решения задачи $D^{L R}(A, b, c)$ с параметрами, приведенными в статье, примененялся квазиньютоновский алгоритм БройденаФлетчера-Гольдфарба-Шанно, что позволило избежать вычисления гессиана.

Исследование выполнено при финансовой поддержке Российского фонда фундаментальных исследований в рамках научного проекта № 18-31-00083

\section{СПИСОК ЛИТЕРАТУРЫ}

1.Еремин, И. И. Введение в теорию линейного и выпуклого программирования / И. И. Еремин, Н. Н. Астафьев. - М. : Наука, 1976. - 192 c.

2. Khachay, M. Yu. On Approximate Algorithms of a Minimal Committee of a Linear Inequalities System // Pattern Recognition and Zimage Analysis. - 2003. - vol. 13, no. 3., pp. 459-464.

3. Еремин, И. И. Несобственные задачи математического программирования / И. И. Еремин, А. А. Ватолин // Проблемы управления и теории информатизации. 1989. - T. 18, №6. - С. 1-23.

4. Ерохин, В. И. Согласование материального баланса крупного нефтеперерабатывающего завода в условиях неполных данных /
В. И. Ерохин, А. Ю. Лаптев, Н. В. Лисицын // Известия Российской академии наук. Теория и системы управления. - 2010. -№ 2. - С. 130140.

5. Скарин, В. Д. О некоторых универсальных методах коррекции несобственных задач выпуклого программирования // Автоматика и телемеханика. - 2012. - № 2. - С. 99-110.

6. Горелик, B. А. Интервальная коррекция непродуктивной матрицы прямых затрат в линейной модели межотраслевого баланса / В. А. Горелик, В. И. Ерохин // Моделирование, оптимизация и декомпозиция сложных динамических процессов. - М. : ВЦ РАН, 2001. C. 51-56.

7. Горелик, В. А. Оптимальная (по минимуму полиэдральной нормы) матричная коррекция несовместных систем линейных алгебраических уравнений и несобственных задач линейного программирования / В. А. Горелик, В. И. Ерохин // Моделирование, декомпозиция и оптимизация сложных динамических процессов. - М : ВЦ РАН, 2004. C. 35-63.

8. Горелик, В. А. Оптимальная матричная коррекция несовместных систем линейных алгебраических уравнений по минимуму евклидовой нормы / В. А. Горелик, В. И. Ерохин. - М. : ВЦ РАН, 2004. - 193 с. 
О матричной коррекции двойственной пары несобственных задач линейного программирования ...

9. Ерохин, В. И. Минимальные по евклидовой норме матричные коррекции задач линейного программирования / В. И. Ерохин, А. С. Красников, М. Н. Хвостов // Автоматика и телемеханика. - 2012. - № 2. - С. 11-24.

10. Попов, Л. Д. Лексикографическая регуляризация и двойственность для несобственных задач линейного программирования / Л. Д. Попов, В. Д. Скарин // Труды института математики и механики УрО РАН. - 2015. T. 21, № 3. - С. 279-291.

11. Ерохин, В. И. Матричная коррекция несобственных задач линейного программирования по минимуму евклидовой нормы с произвольными весами и фиксированными элементами // Математическое программирование: Труды XIII Байкальской международной школы-семинара «Методы оптимизации и их приложения», Иркутск, Байкал 2-8 июля 2005 года. Том I. - Иркутск: ИСЭМ СО РАН, 2005. - C. 105-110.

12. Хвостов, М. Н. О достаточных условиях разрешимости несобственных задач ЛП 1-го рода после матричной коррекции их допустимой области по минимуму взвешенной евклидовой нормы с учетом структурных ограничений // Вестник Воронежского государственного университета. Серия: Физика. Математика. - 2015. - №2. - С. 150-167.

13. Еремин, И. И. Двойственность для несобственных задач математического программирования / И. И. Еремин, А. А. Вато- лин. - Свердловск : ИММ УрО РАН, 1985. $50 \mathrm{c}$.

14. Ерохин, В. И. Матричная коррекция двойственной пары несобственных задач линейного программирования // Журнал вычислительной математики и математической физики. - 2007. - Т.47, № 4. - С. 587- 601.

15. Ерохин, В. И. Матричная коррекция двойственной пары несобственных задач линейного программирования с блочной структурой / В. И. Ерохин, А. С. Красников // Журнал вычислительной математики и математической физики. - 2008. - Т. 48, № 1. - С. 80-89.

16. Волков, В. В. Минимальная по евклидовой норме матричная коррекция пары двойственных задач линейного программирования / В. В. Волков, В. И. Ерохин, А. С. Красников, А. В. Разумов, М. Н. Хвостов // Журнал вычислительной математики и математической физики. - 2017. - Т. 57, № 11. - С. 17881803.

17. Еремин, И. И. Двойственность для несобственных задач линейного программирования // Математические заметки. - 1982, T. 32, № 2, - C. 229-238.

18. Erokhin, V. I. Matrix correction minimal with respect to the Euclidean norm of a pair of dual linear programming problems / V. I. Erokhin, A. S. Krasnikov, V. V. Volkov, M. N. Khvostov // CEUR Workshop Proceedings 9th. 2016. - C. 196-209.

Ерохин В. И. - д-р физ.-мат. наук, профессор, старший научный сотрудник Военно- космической академии имени А. Ф. Можайского. E-mail: erohin_v_i@mail.ru

Волков В. В. - канд. физ.-мат. наук, доцент, доцент кафедры естественнонаучных и общеобразовательных дисциплин Борисоглебского филиала Воронежского государственного университета. E-mail: volkov@bsk.vsu.ru

Хвостов М. Н. - канд. физ.-мат. наук, доцент кафедры естественнонаучных и общеобразовательных дисциплин Борисоглебского филиала Воронежского государственного университета. E-mail: khvostov@bsk.vsu.ru 


\title{
ABOUT MATRIX CORRECTION OF A DUAL PAIR OF IMPROPER LINEAR PROGRAMMING PROBLEMS WITH RESPECT TO THE MINIMUM WEIGHTED EUCLIDEAN NORM
}

\author{
V. I. Erokhin ${ }^{\star}$, V. V. Volkov ${ }^{\star \star}$, M. N. Khvostov ${ }^{\star *}$ \\ ${ }^{*}$ Mozhaisky Military Space Academy,

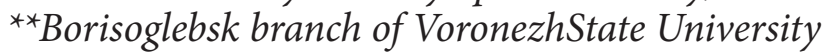

\begin{abstract}
Annotation. The paper focused on problem of matrix correction of a dual pair of improper linear programming problems with respect to the minimum weighted Euclidean norm. Weighted matrix is achieved by multiplying the extended left and right correction matrix by non-degenerate matrices. The main purpose of weighing is to include in the linear programming problem information about the complexity of the correction of the expanded matrix of the constraint system. Matrix correction of linear programming problems is a change (correction) of any coefficients of both left and right parts of equations and inequalities of constraints of primal and dual linear programming problems. The indicated problem is reduced to the auxiliary problem of unconditional differentiable minimization. The theorem about the optimal matrix correction of a dual pair of improper linear programming problems with respect to the minimum weighted Euclidean norm is presented in the article. This theorem is a consequence of the theorem about the existence of a solution to the matrix correction problem of an extended constraint matrix of a dual pair of improper linear programming problems with respect to the minimum weighted Euclidean norm. In turn, the last theorem is based on the theorem about matrix solution for the inverse linear programming problem. The statements of the that theorems are also given in the article. As a possible tool for the numerical solution of this problem, the quasinewton Broyden-Fletcher-Goldfarb-Channo algorithm is considered. The problem of searching for an expanded correction matrix that is minimal in terms of a weighted Euclidean norm is considered. This problem is determined by the following parameters: the expanded matrix of the constraint system, non-degenerate weight matrices, and the initial approximation. The solution is represented by the argument of the objective function and its value. The results of computational experiments of algorithm convergence in terms of the objective function and the argument are given.

Keywords: linear programming, improper linear programming problem, dual pair of linear programming problems, matrix correction, weighted Euclidean norm.
\end{abstract}

Erokhin V. I. - Doctor of physical and mathematical sciences, Professor, Senior researcher of Mozhaisky Military Space Academy. E-mail: erohin_v_i@mail.ru

Volkov V. V. - PhD of physicaland mathematical sciences, Associate Professor of Borisoglebsk branch of VoronezhState University. E-mail: volkov@bsk.vsu.ru

Khvostov M. N. - PhD of physical and mathematical sciences, Associate Professor of Borisoglebsk branch of VoronezhState University. E-mail: khvostov@bsk.vsu.ru 